\title{
The Prevalence of Oral Leukoplakia: Results From a Romanian Medical Center
}

\author{
Ramona Vlad, DMD \\ Department of Odontology and Oral Pathology, Faculty of Dental Medicine, \\ University of Medicine and Pharmacy of Tîrgu Mureș, Romania \\ Irinel Panainte, DMD \\ Department of Orthodontics, Faculty of Dental Medicine, University of \\ Medicine and Pharmacy of Tîrgu Mureș, Romania \\ Alexandra Stoica, Assistant Lecturer, DMD \\ Monica Monea, Associate Prof., DMD, PhD \\ Department of Odontology and Oral Pathology, Faculty of Dental Medicine, \\ University of Medicine and Pharmacy of Tîrgu Mureș, Romania
}

doi: 10.19044/esj.2016.v12n27p12 URL:http://dx.doi.org/10.19044/esj.2016.v12n27p12

\begin{abstract}
The aim of our study is to measure the prevalence of oral leukoplakia in a selected population group in association with variables as gender, age, smoking and alcohol intake. The mucosal lesions were diagnosed and classified according to internationally accepted criteria. Results showed that the majority of oral leukoplakia belonged to homogenous type and only o few were non-homogenous, with a prevalence of 3.32\% 1.10\% respectively, a difference that was statistically significant. The most frequent location of oral leukoplakia was the oral mucosa of the cheek, lower lip, tongue and floor of the oral cavity. Our conclusion was that oral leukoplakia occurred more frequently in men over the age of 45 and smoking and alcohol abuse were positive correlated factors.
\end{abstract}

Keywords: Oral leukoplakia, premalignant oral lesions, white oral lesions

\section{Introduction}

Oral leukoplakia (OL) was defined as a predominantly white lesion or plaque that affects the oral mucosa and cannot be characterized based on clinical or histopathological criteria as any other disease [Kayalvizhi et al 2016]. Worldwide, the prevalence of OL is reported to be between 1\%-2\% for all age groups, with differences in gender distribution and geography [Pelti 2003]. The importance of this condition is represented by the high risk of malignant transformation in squamous carcinoma, evaluated to be 
between 3-17,5\%. The lesions are usually diagnosed after the age of 40 and are six times more frequent among men smokers compared to non-smokers [Baric et al 1982]. Smoking and alcohol abuse might be independent or associated risk factors. There are two types of oral leukoplakia: homogenous and non-homogenous, according to the surface color and morphological characteristics. Homogenous lesions are usually asymptomatic, thin and uniformly flat, while non-homogenous OL are often symptomatic with nodular, verrucous or exophytic aspect [van der Waal et al 2002, Ribeiro et al 2010]. The prevalence of OL reported in the literature ranges from $0.6 \%$ to $4.6 \%$ depending on different factors as study methodology and diagnostic criteria. The information is little information about the incidence of this premalignant lesions in our country but a mean value of $3 \%$ had been previously published. The aim of our study was to measure the prevalence of oral leukoplakia in a group of adult patients referred for dental treatment or routine control to the Department of Odontology and Oral Pathology of our faculty.

\section{Material and methods}

The study was carried out on 271 patients, 156.men and 115 women aged between 26-72 years old, who presented to the department of Odontology and Oral Pathology of the Faculty of Dental Medicine of Tîrgu Mureș for routine treatment or control. All cases of white lesions of the oral mucosa were subjected to detailed examination and those found to be infective or as part of a systemic disease were excluded from the study. The examination of oral mucosa was carried out using artificial light and dental mirrors with magnification and the recording was done based on World Health Organization standards [Kramer et al 1980]. As part of the extensive clinical examination, the presence of OL was recorded by the senior author (MM) who has extensive experience in the diagnosis of oral mucosal lesions. The diagnosis was based on clinical examination and was established after other conditions of oral mucosa that appear as white plaques were excluded and was completed with histopathological evaluation in selected cases. Statistical analysis of data was performed according to Chi-square test.

\section{Results}

The majority of the lesions were homogenous, with a prevalence of $3.32 \%$ for homogenous and $1.10 \%$ for non-homogenous type of lesion, a difference that was statistically significant (Table 1). The most frequent location of OL was the oral mucosa of the cheek 6 cases $(50 \%)$, lower lip 3 cases $(25 \%)$, tongue 2 cases $(16.65 \%)$ and floor of the oral cavity 1 case $(8.35 \%)$. 
Table 1. Incidence of oral leukoplakia in the study group

\begin{tabular}{|c|c|c|}
\hline Type of lesion & Number of lesions & $\%$ \\
\hline Leukoplakia & 12 & 4.42 \\
\hline Homogenous & 9 & 3.32 \\
\hline Non-homogenous & 3 & 1.10 \\
\hline
\end{tabular}

The results of our study showed that the prevalence of OL was 4,42\% in a group of population that was relatively small and selective. OL occurred more frequently in men over 45 years of age (Table 2) especially smokers; the oral mucosa was the most frequent location followed by lower lip.

Table 2. Age distribution of OL in the study group

\begin{tabular}{|c|c|}
\hline Age interval & Incidence of OL \\
\hline$<40$ years & $10,5 \%$ \\
\hline $40-49$ years & $24,5 \%$ \\
\hline $50-59$ years & $19,8 \%$ \\
\hline 60-69 years & $29,4 \%$ \\
\hline Over 70 years & $15,8 \%$ \\
\hline
\end{tabular}

The homogenous type of OL presented with a regular, smooth whitish surface and well-defined margins and the non-homogenous type was associated with an erythematous component or a nodular, ulcerated surface. The lesions were observed in $46,2 \%$ of women and $57,8 \%$ of men and the highest prevalence of oral leukoplakia was found in patients between 60-69 years old who used both tobacco and alcohol. The correlation between OL with known risk factors is presented in table 3.

Table 3. Association between OL and potential risk factors

\begin{tabular}{|c|c|}
\hline $\begin{array}{c}\text { Potential risk factor } \\
\begin{array}{c}\text { Chronic irritation due to caries or dental } \\
\text { restorations }\end{array}\end{array}$ & $5(41,67 \%)$ \\
\hline Poor oral hygiene & $10(83,34 \%)$ \\
\hline Alcohol & $9(75 \%)$ \\
\hline Tobacco & $11(91,67 \%)$ \\
\hline
\end{tabular}

Idiopathic OL were generally benign lesions that occurred in response to local chronic irritation as carious lesions or inadequate dental restorations and were observed in 5 cases $(41,67 \%)$, while tobacco, described as the most important incriminating factor was present in 11 cases $(91,676 \%)$. Another important potential risk factors were poor oral hygiemne and alcohol abuse observed in $83,34 \%$ and $75 \%$ of the cases respectively.

\section{Discussion}

The World Health Organization considered as potentially malignant lesions or disorders the following conditions: erythroplakya, lichen planus, leukoplakia, oral submucous fibrosis and actinic cheilitis. Oral leukoplakya 
is the most common premalignant oral lesion within the oral epithelium with a malignant transformation rate of 5\% according to published data. In a study conducted by Fan et al [Fan et al 2016] the OL was associated with an increased risk of esophageal squamous cell carcinoma. It occurs with a frequency of $0,2-11,7 \%$ at an average age of 60 years old, men being affected three times more frequent than women, although in young patients the proportion is considered to be 1:1 due to frequent smoking among women. OL was defined by van der Waal as " a predominantly white lesion or plaque of a questionable behaviour having excluded clinically and histopathologically any other definable white disease or disorder". The results of our study showed that gender had little influence on incidence of OL, as it was observed in $46,2 \%$ of the women and $56,8 \%$ of male patients. The most affected age groups were between 60-69 and years, which is in accordance to other published data. The etiology of OL is still not fully understood and numerous local (tobacco, alcohol, viral and fungal infections) and systemic predisposing factors (diabetes, hormonal disturbances, hyposalivation, Plummer-Vinson syndrome) might be taken into consideration. The onset is usually observed after the age of 40 and gender distribution ranges from a strong male predominance of 6:1 to almost 1:1 [van de Waal 2002]. Smoking alone or in association with alcohol abuse is considered to be the most important etiological factor in the development of OL and smokers have an increased risk to be diagnosed with this condition compared to non-smokers [Bokor-Bratic, 2000]. Oral leukoplakia is considered a potential malignant disorder with a conversion rate between 0,1\%-17,5\% [Warnakulasuriya 2009, Ho et al 2009, Amagosa et al 2011] and the prevalence of this condition of $4,42 \%$ recorded in our study is relatively high compared to previous evaluations. This could be explained that many patients presented in late stages when the lesions were already under malignant transformation. We should emphasize the importance of associated risk factors as tobacco, alcohol or other inflammatory conditions as periodontal disease, that could contribute to the development of oral leukoplakia. In order to have a uniform reporting system for the prevalence of OL, the clinical type, size, and histopathologic aspect of the lesion together with gender, age at diagnostic moment and associated risk factors must be recorded. Early detection of oral premalignant lesions such as OL that might transform into oral cancer by screening methods is extremly important. Saliva as a diagnostic fluid seem to be promising and has a number of advantages when compared to the blood testing. Histopathological diagnosis is still considered the golden standard in the case of oral premalignant lesions but studies on salivary citokines showed promising results. 


\section{Conclusion}

The results of our study showed that the prevalence of oral leukoplakia was 4,42\%, mostly in patients over 60 years of age with relatively equal distribution between sexes, in which smoking and alcohol abuse were identified as routine habits. The majority of the lesions were homogenous, with a prevalence of $3.32 \%$ for homogenous and $1.10 \%$ for non-homogenous type of lesion, a difference that was statistically significant and the most frequent locations of OL were the oral mucosa of the cheek, lower lip, tongue and the floor of the oral cavity. Due to the possibility of malignant transformation of oral leukoplakia, a better preventive program consisting of careful examination and treatment of any inflammatory conditions of oral mucosa and periodontal tissues must be carried out in order to reduce the incidence of oral cancer.

\section{Aknowledgements}

This study was conducted under the framework of the research grant no. 3688/01.04.2016, financed by SC COSAMEXT SRL Tîrgu Mureș and developed by the University of Medicine and Pharmacy of Tîrgu Mureș.

\section{References:}

Kayalvizhi E.B., Lakshman V.L., Sitra G, Yoga S, Kanmani R, Megalai N. Oral leukoplakia: A review and its update. J of Med Radiol Pathol and Surgery; 2016 (2): 18-22.

Pelti S. Pooled estimate of world leukoplakia prevalence: a systematic review. Oral Oncol; 2003 (39): 770-780.

Baric J.M., Alman J.E., Feldman R.S., Chauncey H.H. Influence of cigarette, pipe and cigar smoking, removal partial dentures and age on oral leukoplakia. Oral Surg Oral Med Oral Pathol; 1982 (54): 424-429.

van der Waal I., Axell T. Oral leukoplakia: o proposal for uniform reporting. Oral Oncol; 2002 (38): 521-526.

Ribeiro A.S., Salles P.R., da Silva T.A., Masquita R.A.. A review of the nonsurgical treatment of oral leukoplakia. Int J Dent; 2010; 2010:186018.

Kramer I.R., Pindborg J.J., Bezroukov V., Infirri J.S.. Guide to epidemiology and diagnosis of oral mucosal disease and conditions. World Health Organization. Community Dent Oral Epidemiol; 1980 (8): 1-26.

Fan J.H., Wang J.B., Qu C.X. et al. Association between oral leukoplakia and upper gastrointestinal cancers: a 28-years follow-up study in the Liuxian general population trial. Oral Oncol; 2014; 50(10): 971-975.

Bokor-Bratic M. The prevalence of precancerous lesions. Oral leukoplakia. Archive of Oncology; 2000 (8): 164-170.

Warnakulasuriya S. Global epidemiology of oral and oropharyngeal cancer. Oral Oncol; 2009 (45): 309-336. 
Ho P.S., Chen P.L., Warnakularusiya S., Shien T.Y., Chen Y.K., Huang I.Y.. Malignant transformation of oral potentially malignant disorders in males: a retrospective cohort study. BMC Cancer; 2009 (9): 260-10.1186/1471-24079-260.

Amagosa T., Yamashiro M., Uzama N. Oral premalignant lesions: from a clinical perspective. Int J Clin Oncol; 2011 (16): 5-14. 